\title{
SIMULATION OPTIMISATION FOR IMPROVING THE EFFICIENCY OF A PRODUCTION LINE
}

\author{
Mr. Joseph Calverley \\ Dr Christine Currie \\ Dr Bhakti Stephan Onggo \\ University of Southampton \\ Highfield Road \\ Southampton \\ SO17 1BJ \\ jac1g17@ soton.ac.uk \\ christine.currie@soton.ac.uk \\ B.S.S.Onggo@soton.ac.uk
}

\author{
Dr. Thomas Monks \\ University of Exeter Medical School \\ Heavitree Road \\ Exeter \\ EX1 1TE \\ t.m.w.monks@exeter.ac.uk
}

\author{
Mr. Michael Higgins \\ PowerTrain Manufacturing Engineering \\ Ford Motor Company \\ Dunton Research Centre \\ Basildon \\ SS15 6EE \\ mhiggi37@ford.com
}

\begin{abstract}
Finding the best set up for a production line is a traditional simulation problem. Here, we apply and adapt Optimal Computing Budget Allocation (OCBA), a well-known method for optimisation via simulation to find the best design for a production line. Typically OCBA is implemented in a sequential fashion with the results of one (or a small number) of replications being used to adapt suggest how the sampling should be allocated in the next step. In this paper we change that format to fit in with the typical experimental set up at Ford and instead work in five main stages. Each stage is allocated a set amount of simulation time and we use OCBA to determine how long to run the simulation for with each system configuration. The results show that using OCBA can substantially increase the efficiency of selecting the best out of a number of designs.
\end{abstract}

\section{Keywords:}

Optimisation via simulation, production line, simulation

\section{INTRODUCTION}

Simulation models are frequently built with the aim of choosing between a number of different system designs and this is the situation we consider here. When there are a large number of designs to choose 


\section{Calverley, Currie, Monks, Onggo, and Higgins}

between and/or the runtime of the simulation model is very long, using methods that optimally allocate simulation time to the different designs can have a significant impact on the efficiency of the experimentation. This is especially important when optimising in real time using a digital twin or symbiotic simulation (Xu et al. 2016).

Ford are keen to implement efficient simulation experimentation to both increase the productivity of their simulation group and move to implementation within a symbiotic simulation. Here, we define a symbiotic simulation to be a model that is automatically fed with data from the real system, and is used to test out different system configurations. We assume in this case that the change in configuration needs to be carried out regularly, and that this kind of problem is encountered in, for example, job shop production systems and flexible manufacturing systems. Our definition of symbiotic simulation in this paper is very similar to the digital twin idea but with a focus on developing optimisation methods that are carried out frequently during production (and hence need to be computationally efficient) rather than simply replicating the system and forecasting future behaviour.

Optimisation via simulation (OvS) methods aim to find either a single optimal solution or a set of optimal solutions using stochastic simulation for evaluation. Solving these problems is difficult because of the randomness of the simulation output and OvS is an active field of research (see (Fu 2015) for an overview).

The problem we consider here is described as ranking and selection, where we are choosing between a finite number of discrete options. Branke et al. (2007) classify ranking and selection algorithms into three categories: (i) the indifference zone approach; (ii) expected value of information procedures (VIP) and (iii) optimal computing budget allocation (OCBA). Indifference zone approaches such as Kim and Nelson (2006) aim to guarantee a probability of correct selection (PCS) of the optimal configuration while OCBA and VIP procedures look for the best allocation of a finite simulation budget. The latter philosophy fits much better with the implementation of simulation optimisation within a symbiotic simulation and we choose to adapt OCBA in what follows.

In the remainder of the paper we introduce OCBA (Section 2), before going on to formulate the problem (Section 3) and describe the methodology (Section 4). We implemented the optimisation routine on a case study from a manufacturing line and we describe this in Section 5 alongside some preliminary results.

\section{OCBA}

For readers with a particular interest in finding out more about OCBA, there is an excellent book by Chung-Hun Chen and Loo Hay Lee that describes its use (Chen and Lee 2010). Here, we give an overview of how it works.

OCBA is a heuristic method that aims to efficiently allocate simulation time to each of a number of competing designs. Its basic premise was first introduced in the 1990s (Chen 1996) and is centred around the fact that allocating more simulation time to a design allows a more precise answer to be obtained about its output. As a result, OCBA will tend to allocate more time to designs which are critical to the decision over which is most important; for example, designs which are definitely not contenders for the best will be allocated little simulation time.

Sequential OCBA algorithms will allocate the next simulation replication to the design that most increases the estimated approximate probability of correct selection (EAPCS) relative to the current estimate of the probability of correct selection (PCS) in each time step. Improvements to the efficiency of the initial OCBA heuristic have mainly resulted from refining the assumptions made when estimating EAPCS, allowing a better estimate of how allocating more simulation time to a design will help to solve the problem.

OCBA begins by simulating each design a limited number of times, to obtain information about their means and variances. OCBA then determines the most critical designs, those which are both close to being the best and/or highly variable, and assigns further simulations to these critical designs, until the full computing budget is realized. Simpler sampling methods, such as Equal Allocation (equal number of observations of each system) and Proportional to Variance (allocate more observations to systems with 


\section{Calverley, Currie, Monks, Onggo, and Higgins}

a higher variance) are highly inefficient. Given a limited computing budget, these algorithms will fail to consistently find the true best.

We assume that we are choosing between $k$ designs and have a computational budget of $T$, meaning that we can run a maximum of $T$ replications during the experiment. Note that for simplicity we assume each replication involves the same amount of computational effort. In the most general OCBA algorithm that we describe below, we assume that a total of $\Delta$ replications are allocated at each step of the algorithm. As we run $n_{0} \geq 5$ replications for each of the $k$ designs during the initialisation phase, $T-k n_{0}$ must be a multiple of $\Delta$. Algorithm 1 describes the basic process of OCBA where we aim to maximise the PCS for a fixed number of replications.

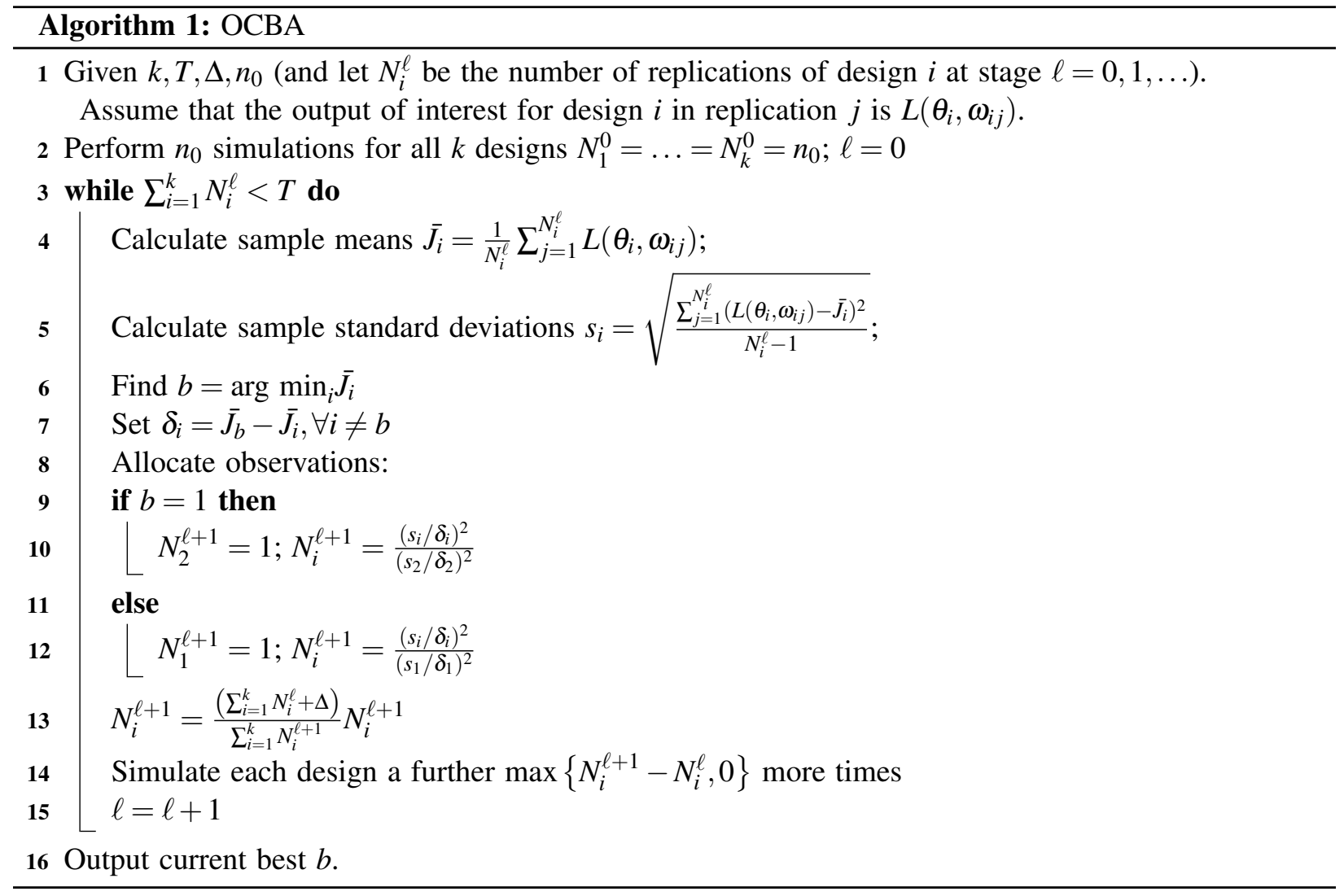

\section{PROBLEM FORMULATION}

We consider the minimisation of the time engines spend on a production line, so-called dock-to-dock times. A set of 15 different system designs are considered. What is particularly interesting for this article is that Ford place a restriction of 5 on the number of replications that can be run for each design during a simulation experiment. However, the length of each of those replications can be varied independently. In the example considered in Section 5, we measure the length of a replication by the number of engines that enter the simulation, using this as a proxy for simulation duration.

By restricting the number of replications to 5 but allowing variability in runtime, we first need to rephrase the OCBA algorithm to become one of allocating simulation durations to different designs rather than allocating simulation replications. This is a relatively straightforward change but raises an interesting question of how much total duration we should allocate to each of the 5 replications, which we discuss in the following section. 


\section{METHODOLOGY}

We provide an overview of the method in Algorithm 2.

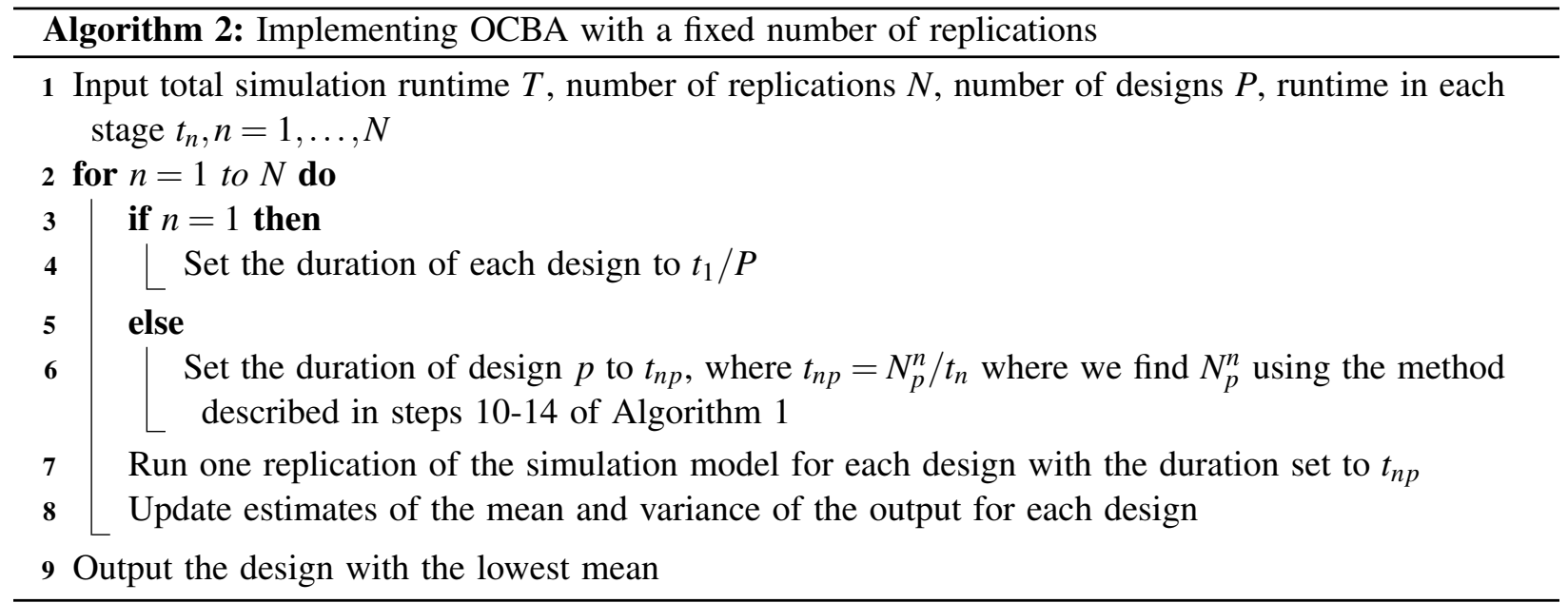

As we show below in the results, we can improve the efficiency of the algorithm through a careful choice of the allocation of runtime to each replication, $t_{n}, n=1, \ldots N$, where $N=5$ for the Ford example. Intuition suggests that setting the $t_{n}$ such that $t_{1}<t_{2}<\ldots<t_{N}$ is likely to result in a more efficient allocation because we spend longer on replications where we have a better understanding of which designs are critical to making the correct selection. In the case study, we test setting $t_{n}=\frac{n^{q}}{\sum_{n=1}^{N} n^{q}} T$ for $q$ in the range $[1,2]$.

\section{CASE STUDY}

When investigating the method, we resample from simulation output that has previously been produced by a Ford simulation model. When implementing this method in practice, the algorithm would instead be used to determine the runtime of each design in each replication. We compare 15 different designs and the simulation data we resample from consists of 75 data files, each containing data from one replication for one design, excluding the warm up period. Each file contains approximately 65,000 data points.

Figure 1 displays a box plot of the results for each design, using all of the simulated replications. This shows that designs 3,11 and 12 have very similar mean dock-to-dock times.

\subsection{Initial Analysis and Checks}

We carry out two statistical checks on the data before beginning the optimisation.

\section{- Check for autocorrelation in the output}

As the simulation data are in the form of a time series, there is a possibility that earlier data points can influence the values of later data points, a phenomenon described as autocorrelation. If this is present then instead of using the raw data, it is necessary to batch adjacent points together and use the means of these batches as our input data to the optimisation algorithm. For the data set we consider here, there is no evidence of autocorrelation in any of the data files; therefore we have not used batching.

- Check that the output follows a normal distribution

The method underlying OCBA assumes that outputs follow a normal distribution. If the data are not normal, the approximations of the expected improvements in the probability of correct selection are less accurate and the convergence results no longer hold. Nonetheless, the algorithm is still expected to provide a good guide of how to allocate observations. We find that none of the data follows a normal distribution. 


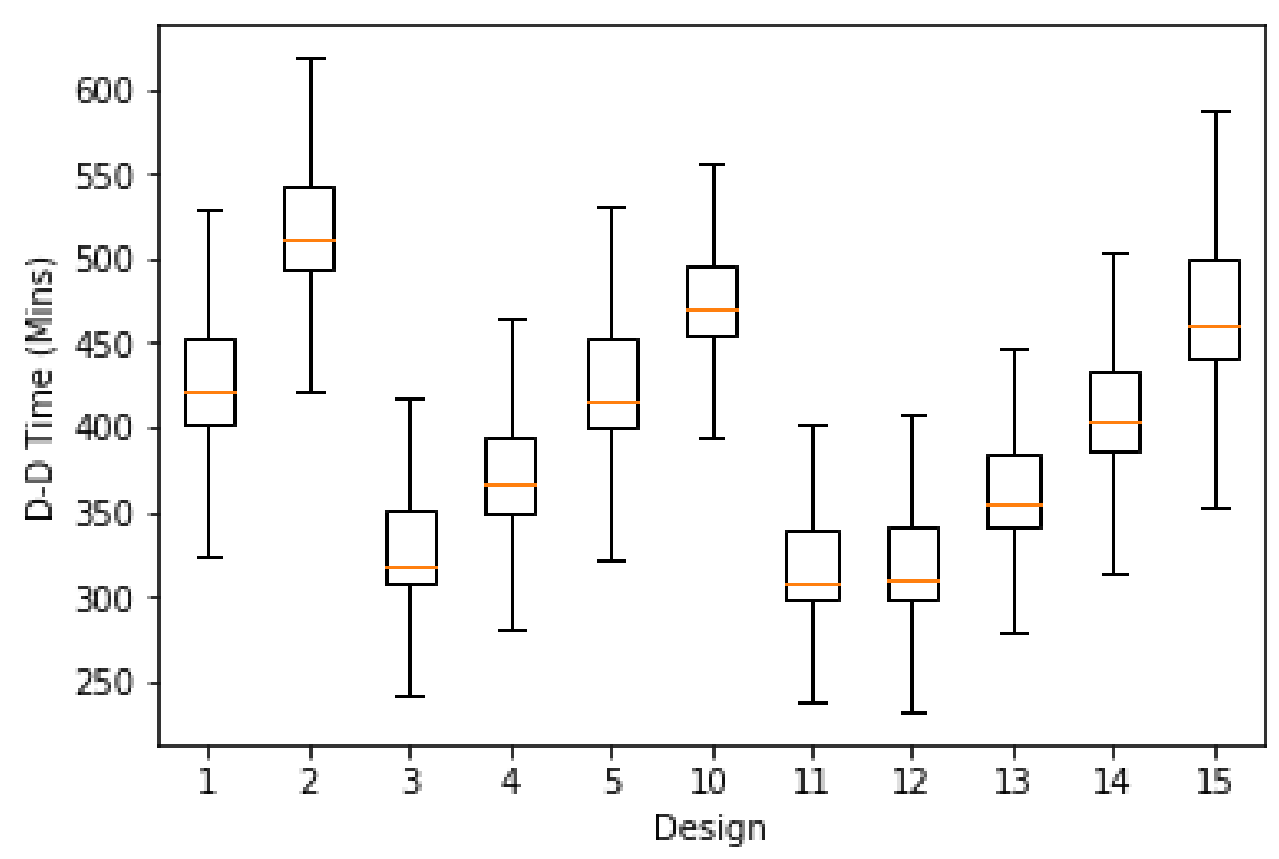

Figure 1: Box Plot of the Dock to Dock Times for each Design. Values are taken from all replications.

\subsection{Implementing OCBA-PCS}

OCBA-PCS aims to maximise the Probability of Correct Selection PCS. As a baseline, we test the method using the standard sequential algorithm (rather than with limited replications), deciding which design to sample next at each timestep. The number of timesteps in the first stage is $t_{1}=5 P, 5$ per design; and the simulation budget $T=11,000$. We ran the method 100,000 times, selecting the correct design $95.37 \%$ of the time, using approximately $0.003 \%$ of the total data points provided. We use this as the baseline for comparison with our method using the Ford set up of 5 replications. Figure 2 describes how the sequential OCBA algorithm works.

When implementing OCBA across 5 stages, we first consider how to allocate the runtime across the different stages such that the ratio of runtimes is $1^{p}: 2^{p}: 3^{p}: 4^{p}: 5^{p}$ across the 5 stages. We measure PCS where $T=5000$ for different values of $p$ and preliminary results are presented in Figure 3 .

Preliminary results suggest that the algorithm with 5 replications performs well and significantly reduces the number of observations Ford need to make decisions about the structure of their lines.

\section{CONCLUSION}

Preliminary results presented here suggest that when using a small number of replications but allowing the runtime to vary between each design, it is possible to implement OCBA effectively. This has the potential to drastically reduce the duration of experiments and in the Ford example, we used less than $0.003 \%$ of the original simulation output data. This makes the simulation optimisation feasible to incorporate in a symbiotic simulation.

There are several avenues for future research. While maintaining the structure implemented by Ford in which we vary the runtime per replication rather than the number of replications, one consideration is the optimal number of replications. Initial numerical results suggest that 5 replications works well but we need to perform a proper comparison with a full sequential algorithm to better gauge the quality of its performance. 


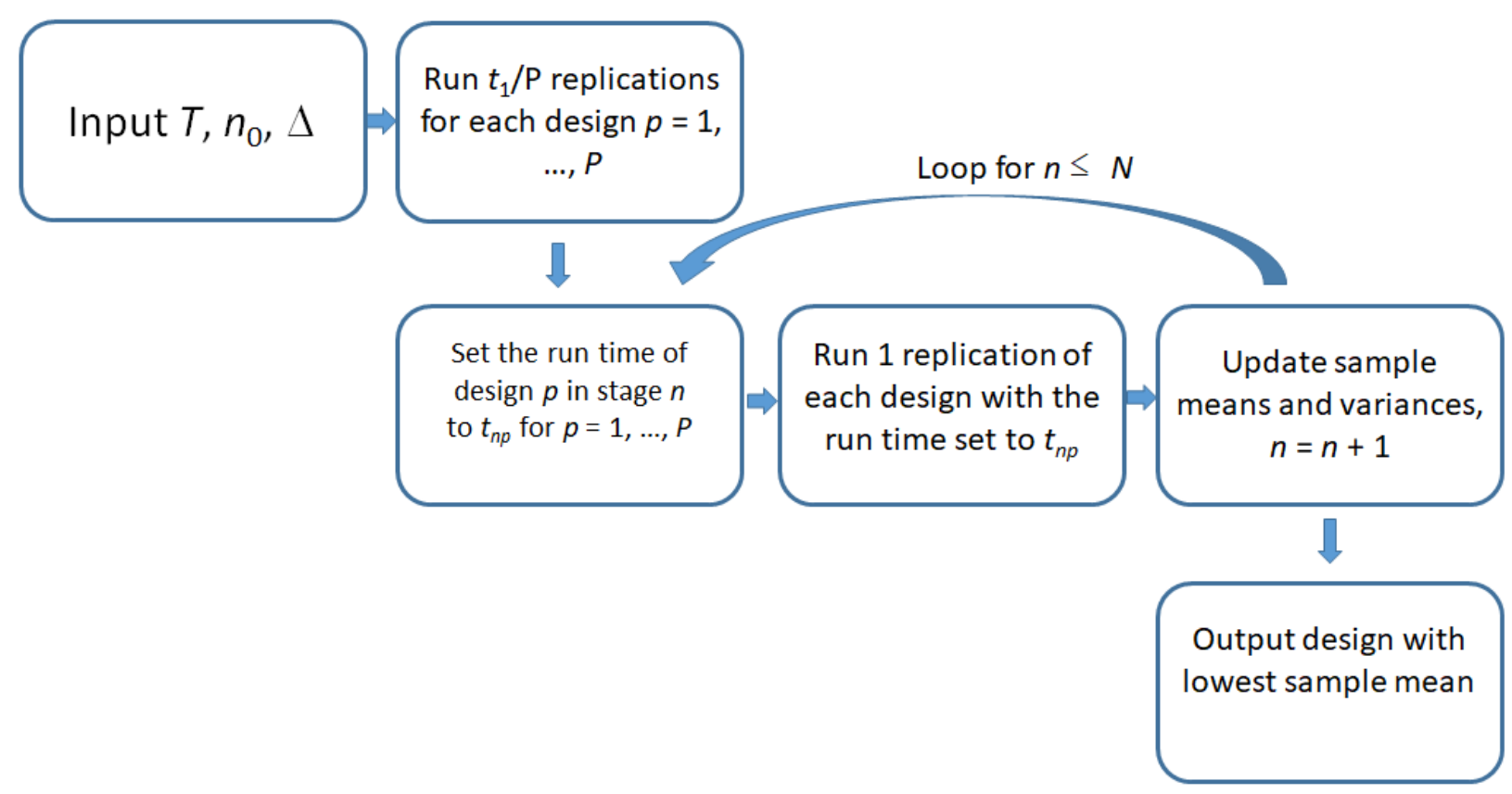

Figure 2: Graphic representation of OCBA based on PCS

In the work we describe here, we aim to maximise the probability of correct selection but a stream of research exists in which the aim is instead to minimise the Expected Opportunity Cost (EOC). PCS methods know only if a design is wrong but EOC methods provide a measure of the penalty in making an incorrect selection. The EOC idea was first introduced by (Chick and Wu 2005) and has an intuitive appeal for problems such as this because it directly relates to the economics.

Applying these ideas to other data sets would also be beneficial to determine whether the conclusions hold in other situations.

\section{ACKNOWLEDGMENTS}

Joseph Calverley was funded by an EPSRC vacation grant at the University of Southampton (EP/R513325/1).

\section{REFERENCES}

Branke, J., S. E. Chick, and C. Schmidt. 2007. "Selecting a Selection Procedure". Management Science 53:1916-1932.

Chen, C.-H. 1996. "A Lower Bound for the Correct Subset-Selection Probability and its Application to Discrete Event Simulations.". IEEE Transactions on Automatic Control 41:1227-1231.

Chen, C.-H., and L. H. Lee. 2010, 07. Stochastic Simulation Optimization: An Optimal Computing Budget Allocation.

Chick, S., and Y. Wu. 2005. "Selection procedures with frequentist expected opportunity cost bounds". Operations Research 53:867-878.

Fu, M. C. 2015. Handbook of Simulation Optimization. 1st ed. New York, USA: Springer.

Kim, S.-H., and B. L. Nelson. 2006. "Selecting the Best System". In Elsevier Handbooks in Operations Research and Management Science: Simulation, edited by S. G. Henderson and B. Nelson. Waltham, MA: Elsevier.

Xu, J., E. Huang, L. Hsieh, L. H. Lee, Q.-S. Jia, and C.-H. Chen. 2016. "Simulation optimization in the era of Industrial 4.0 and the Industrial Internet". Journal of Simulation 10 (4): 310-320. 


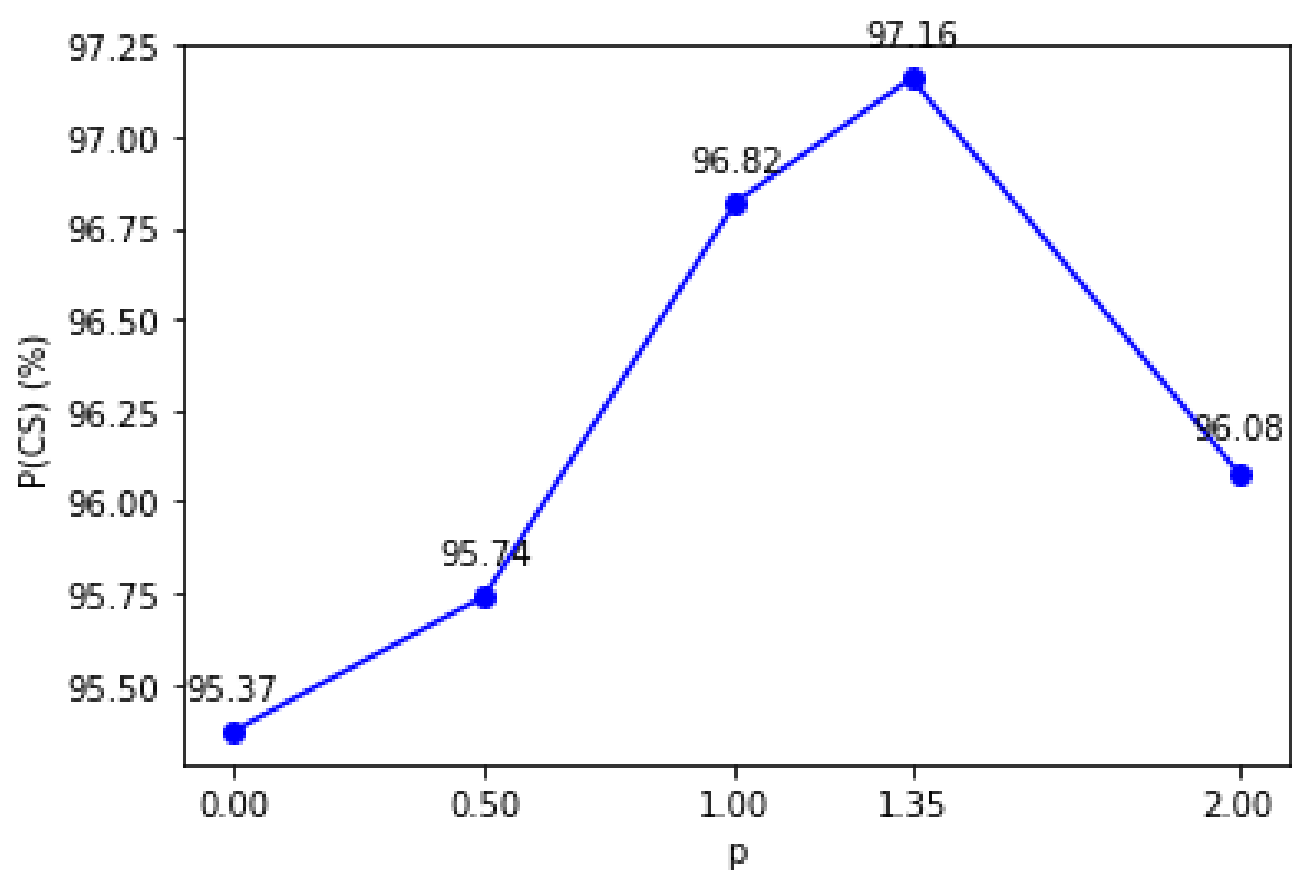

Figure 3: Effect of change in $p$ on $\mathrm{P}(\mathrm{CS})$

\section{AUTHOR BIOGRAPHIES}

JOSEPH CALVERLEY is an undergraduate student at the University of Southampton studying for a BSc in Mathematics, Operational Research, Statistics and Economics. He worked on this project during his summer vacation.

CHRISTINE CURRIE is Associate Professor of Operational Research in Mathematical Sciences at the University of Southampton, UK, where she also obtained her Ph.D. She is Editor-in-Chief for the Journal of Simulation. Christine was co-chair of the Simulation Special Interest Group in the UK Operational Research Society until September 2013. Her research interests include simulation optimisation, mathematical modelling of epidemics, optimal pricing and applications of simulation in health care. See http://www.southampton.ac.uk/maths/about/staff/ccurrie.page for more details.

THOMAS MONKS is an Associate Professor of Health Data Science at the University of Exeter Medical School and The Institute of Data Science and AI, University of Exeter. He is also Turing Fellow at The Alan Turing Institute and Honorary researcher at the Clinical Operational Research Unit, UCL. Between 2014 and 2019 he led an applied healthcare Data Science team funded by the NIHR. He has worked as both a Software Engineer in the private sector and an Operational Research Analyst within the public sector. His speciality is simulation of healthcare systems of unscheduled and emergency care. He was co-chair of the UKs Simulation Workshop 2016 and 2018. His email is t.m.w.monks@exeter.ac.uk webpage is: https://www.turing.ac.uk/people/researchers/thomas-monks

STEPHAN ONGGO is an Associate Professor of Business Analytics at Southampton Business School, the University of Southampton. He is a member of the Centre of Operational Research, Management Sciences and Information Systems (CORMSIS). His research interests lie in the areas of simulation modeling methodology (symbiotic simulation, hybrid modeling, agent-based simulation, discrete-event simulation) with applications in operations and supply chain management and health care. He is the associate editor for the Journal of 
Simulation and the chair of The OR Societys Simulation SIG. His website is https://bsonggo.wordpress.com

MICHAEL HIGGINS graduated with a Bachelor of Science in Mathematics and Physics in 2011 and in 2014 gained an MPhil (Eng) through his research into the application of Discrete Event Simulation within the automotive industry. He has worked with Ford Motor Company since 2012, where his current role is within PowerTrain Manufacturing Engineering (PTME) as Lead Simulation Engineer. He has gained a holistic view of powertrain manufacturing and has utilized simulation as a tool across several functions within PTME including plant and production engineering roles. He is currently researching and developing methods to increase usability and widen the application of the Ford Simulation Toolset. His email address is mhiggi37@ford.com. 